\title{
Improved linearity using harmonic error rejection in a full-field range imaging system
}

\author{
Andrew D. Payne*a Adrian A. Dorrington $^{\mathrm{a}}$, Michael J. Cree ${ }^{\mathrm{a}}$, Dale A. Carnegie ${ }^{\mathrm{b}}$ \\ ${ }^{a}$ Dept. Engineering, University of Waikato, Private Bag 3105, Hamilton, New Zealand; \\ ${ }^{b}$ Dept. Physical and Chemical Sciences, Victoria University of Wellington \\ PO Box 600, Wellington, New Zealand
}

\begin{abstract}
Full field range imaging cameras are used to simultaneously measure the distance for every pixel in a given scene using an intensity modulated illumination source and a gain modulated receiver array. The light is reflected from an object in the scene, and the modulation envelope experiences a phase shift proportional to the target distance. Ideally the waveforms are sinusoidal, allowing the phase, and hence object range, to be determined from four measurements using an arctangent function. In practice these waveforms are often not perfectly sinusoidal, and in some cases square waveforms are instead used to simplify the electronic drive requirements. The waveforms therefore commonly contain odd harmonics which contribute a nonlinear error to the phase determination, and therefore an error in the range measurement. We have developed a unique sampling method to cancel the effect of these harmonics, with the results showing an order of magnitude improvement in the measurement linearity without the need for calibration or lookup tables, while the acquisition time remains unchanged. The technique can be applied to existing range imaging systems without having to change or modify the complex illumination or sensor systems, instead only requiring a change to the signal generation and timing electronics.
\end{abstract}

Keywords: Phase detection, harmonic rejection, harmonic cancellation, aliasing, linearity, range imaging, 3D camera

\section{INTRODUCTION}

A number of full field image ranging systems, which are capable of simultaneously measuring the distance for every pixel in a given scene, are being developed by various groups ${ }^{1-10}$. These "3D cameras" are suitable for a wide range of applications such as automated factory processes, automotive safety and control, surveying, multimedia integration, and shape reconstruction. Measuring the entire scene in parallel, rather than serially scanning the collimated laser beam of a single point range-finder, allows a much higher data acquisition rate and does not require moving parts (which can be sensitive to vibration).

The sensor arrays used, typically a gain-modulated intensified CCD (ICCD) camera ${ }^{1-5}$ or custom built CMOS/CCD array $^{6-10}$, output data at a similar speed to conventional video cameras - typically 30 frames per second. The object distance is temporally encoded into the intensity of the recorded data, so multiple frames are required to determine range within the scene. Reducing the required number of image frames increases the range data acquisition rate, which is particularly important in a dynamic scene where objects, or the camera, may be moving. However, when using a small number of data points to encode/decode the object range, systematic errors can have significant impact on the results.

A method to significantly reduce systematic error caused by signal harmonics is presented here. Although theoretically the error can be calibrated out in a traditional system, this incurs additional time and expense, particularly if system parameters are likely to change. Utilizing the method described here, we demonstrate up to an order of magnitude improvement in the uncalibrated range precision.

\section{PRINCIPLE OF OPERATION OF A FULL-FIELD IMAGE RANGER}

A light source is intensity modulated at a high frequency, usually between $10-100 \mathrm{MHz}$, and illuminates the field of view. Objects within the field reflect the light back towards a lens which images the light onto a receiver array. Due to the round-trip time, the phase angle $\varphi$ of the light source modulation envelope is retarded as given by equation (1), where

*adp2@waikato.ac.nz

Three-Dimensional Image Capture and Applications 2008, edited by Brian D. Corner, Masaaki Mochimaru, Robert Sitnik Proc. of SPIE-IS\&T Electronic Imaging, SPIE Vol. 6805, 68050D, @ 2008 SPIE-IS\&T · 0277-786X/08/\$18 
$f_{\text {mod }}$ is the modulation frequency, $d$ is the distance to the object, and $c$ is the speed of light.

$$
\varphi=\frac{4 \pi f_{\bmod } d}{c}
$$

The receiver array synchronously samples the incoming reflected optical signal. Typically four samples $\mathrm{A}_{0}-\mathrm{A}_{3}$ per modulation period $T_{\text {mod }}$ are used ${ }^{5-10}$ as shown in figure 1.

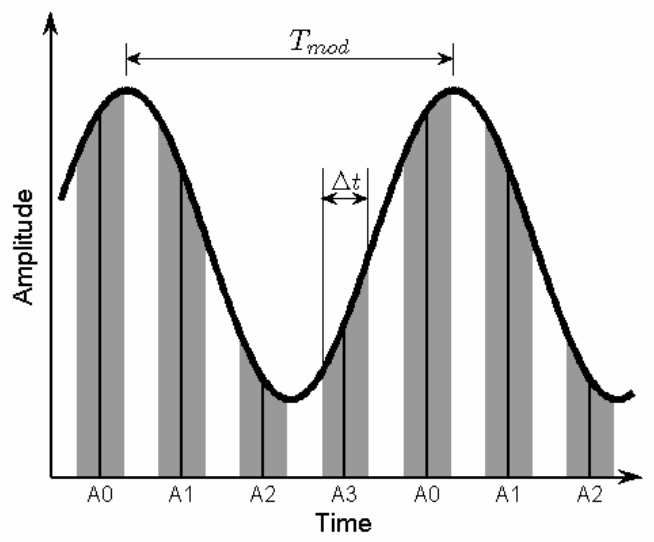

Fig. 1. Synchronous sampling of the reflected sinusoidal intensity modulated waveform.

The four measurements allow the three unknown variables to be determined ${ }^{7,9}$, namely the signal amplitude $A$ and phase $\varphi$, and the background illumination $B$ :

$$
\begin{gathered}
A=\frac{\sqrt{\left(A_{0}-A_{2}\right)^{2}+\left(A_{1}-A_{3}\right)^{2}}}{2} \\
B=\frac{A_{0}+A_{1}+A_{2}+A_{3}}{4} \\
\varphi=\tan ^{-1}\left(\frac{A_{0}-A_{2}}{A_{1}-A_{3}}\right) .
\end{gathered}
$$

By measuring the phase angle of the modulation envelope using equation (4), substituting into equation (1) and rearranging, the distance $d$ to each object in the scene can be determined. A diverging light source and a receiver array allow all points in the scene to be acquired simultaneously, producing extremely fast range acquisition for applications requiring a high resolution range image.

\section{WORKING WITH FOUR DATA POINTS}

Some image ranging systems capture two data frames to determine the scene range ${ }^{1-3}$, either by sampling the modulation waveform at $0^{\circ}$ and $180^{\circ}$, or by sampling at $0^{\circ}$ and turning off the light source for the second frame. Speed is maximized for these systems; however it is not possible to uniquely determine all three dynamic variables, namely the signal amplitude, signal phase, and the background light amplitude. Optical bandpass filters centred at the emitted wavelength, and control of the wavelengths of surrounding light sources, allows the two remaining variables to be uniquely determined; however this constrains the operating environment, for example to a studio ${ }^{3}$. Acquiring three data frames ${ }^{4}$ allows all three variables to be uniquely solved, however it is often more efficient to capture four frames to determine the range, especially if a multi-tap custom sensor is used ${ }^{6-10}$. As this is the most common method employed we will consider only this method for the remainder of this paper.

The aforementioned method is suited to sinusoidal intensity modulation of the light source, where only one fundamental frequency exists. In practice, a simplified electronic switching circuit can be utilized to produce square-wave 
modulation, thereby reducing circuit size and complexity, and increasing electrical efficiency. The actual response usually falls somewhere between a square-wave and a sine-wave due to circuit bandwidth limitations, creating a signal containing a number of harmonics. Systems which do attempt to operate with sinusoidal modulation can also unintentionally introduce higher order harmonics due to non-linearity of the components used.

A frequency spectrum of a square-wave signal, showing the fundamental frequency and the first few harmonics, is given in Fig 2. Harmonics which occur above the Nyquist frequency are aliased down to a lower baseband frequency. For a given signal frequency $f_{\text {sig }}$, and sampling frequency $f_{\text {samp }}$, the harmonics which are aliased onto the fundamental frequency are given by

$$
f=i f_{\text {samp }} \pm f_{\text {sig }}
$$

where $i$ is an integer $1,2, \ldots$ With a synchronized sampling rate of $f_{\text {samp }}=4 f_{\text {sig }}$, all of the odd order harmonics are aliased onto the fundamental frequency as illustrated in figure 2 .

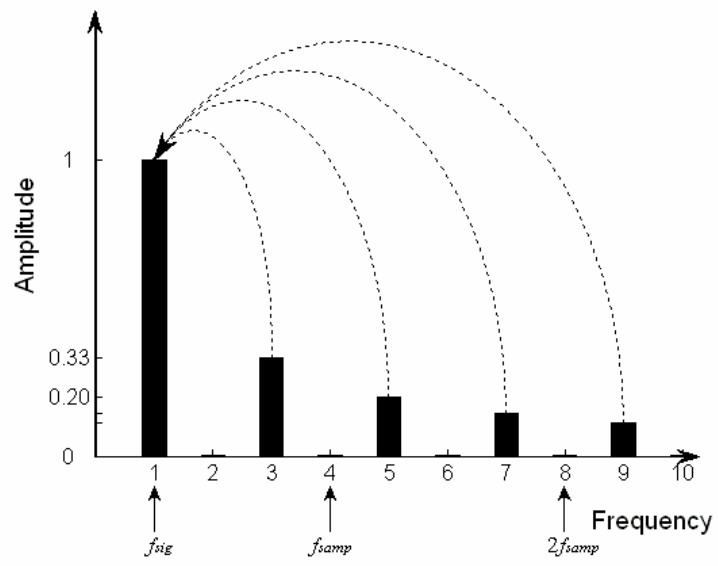

Fig. 2. Odd harmonics of a square wave are aliased onto the fundamental frequency when four data points are acquired.

In other electronic applications a low-pass filter is added to the input before the signal is sampled, rejecting frequencies above the Nyquist frequency (anti-aliasing filter). In our case the receiver is sampling an optical input and therefore cannot be filtered in this manner. The addition of harmonics onto the sampled fundamental frequency causes an error in the phase angle calculation when using equation (4), hence an error in the measured range to an object.

In practice, the input waveform sampling occurs over a window period $\Delta t$ at each of the shaded regions labeled $\mathrm{A}_{0}, \ldots, \mathrm{A}_{3}$ in figure 1. This is achieved by modulating the receiver gain synchronously at the same frequency as the light source modulation, and integrating the output over a large number of modulation periods. The next frame repeats the process, but the modulation of the receiver gain is shifted by $90^{\circ}$. The period $\Delta t$ results in a reduction of the original amplitude $A$ by a magnitude of

$$
\operatorname{sinc}\left(\frac{\pi \Delta t}{T_{m o d}}\right)
$$

where $T_{\bmod }$ is the input waveform modulation period. Receiver gain modulation with a $50 \%$ duty cycle square-wave $\left(\Delta t / T_{\text {mod }}=1 / 2\right)$ is typical for optimal performance ${ }^{7}$ and reduces the fundamental signal amplitude to $64 \%$ of the true value. This sampling window does not alter the phase of the measured signal; hence the range measurement remains valid. Amplitude reduction of the third harmonic is more pronounced as $T_{\text {mod }}$ is reduced by a factor of 3 for the harmonic, but $\Delta t$ remains constant $\left(\Delta t / T_{\bmod }=3 / 2\right)$, reducing the harmonic amplitude to $21 \%$ of its original value. This amplitude reduction continues for all harmonics, hence if both the light source and receiver gain modulation are square waves, the mixing process results in a triangular wave.

To illustrate the error introduced due to harmonics, a simulation was performed by creating an approximation to a triangular waveform by adding frequency components up to the ninth order and sampling it at four points separated by 
$90^{\circ}$ for various input phase angles. The phase of the fundamental component was calculated using equation (4), and the result plotted in figure 3. The resultant error has a distinctive cyclic nature, and the peak to peak error amplitude is approximately $150 \mathrm{mrad}$. Four cycles occurring over a $2 \pi$ period result from the third and fifth order harmonics. It is worth noting that even numbered harmonics were therefore not included in the simulation because they do not contribute any error in the recovered phase as they are not aliased onto the fundamental frequency during sampling (refer figure 2). Furthermore, in the case of perfect square-wave modulation, even harmonics are not present.
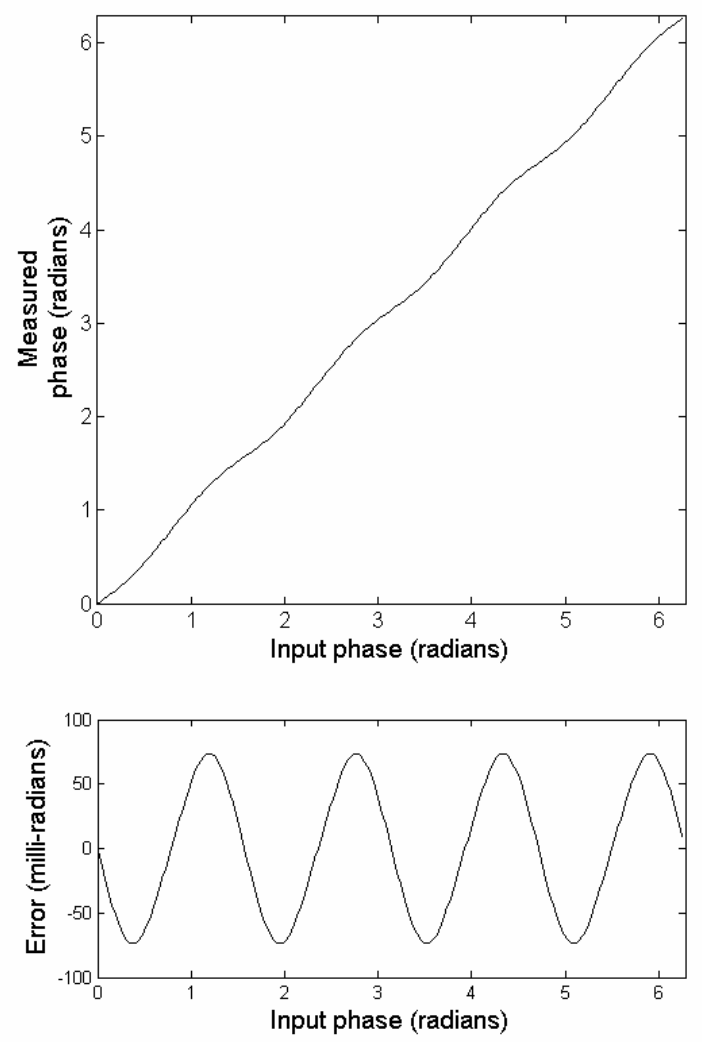

Fig. 3. Phase error incurred using equation (4) with a resultant triangle wave, where the illumination and receiver modulation waveforms are square.

This cyclic error is visible in experimental results presented by other groups ${ }^{11,12}$. The range errors are analysed in detail by Kahlmann and Ingensand ${ }^{12}$, and the peak-to-peak cyclic range errors can be seen to be approximately $10-15 \mathrm{~cm}$, equivalent to $80-125 \mathrm{mrad}$ (calculated using equation (1), using $f_{\text {mod }}=20 \mathrm{MHz}$ for their system). This error closely matches the amplitude and shape of the waveform in figure 3. The measurement precision reported by Kahlmann and Ingensand ${ }^{12}$ is $6 \mathrm{~mm}$ in the centre of the image, an order of magnitude smaller than the cyclic error; hence harmonic error is a significant contributor to the accuracy of the system.

\section{DEALING WITH HARMONICS}

To influence the phase measurement each harmonic must be present in both the light source waveform and receiver gain modulation waveform. If one of these waveforms is reduced to a sinusoid, the error will not be present. Due to the frequencies involved (typically $20-44 \mathrm{MHz}^{7-10}$ ) and the bandwidth limits of CMOS/CCD receiver arrays and LEDs, this can be a demanding requirement.

For a typical modulation waveform the harmonic amplitude decreases rapidly with increasing order. By taking a large number of samples, separated by an equal phase step, $f_{\text {samp }}$ in equation (5) is increased. The harmonic frequencies which are aliased down to the fundamental frequency now have significantly smaller amplitude, thus result in smaller errors. The additional data acquisition time required limits the usefulness of this method for dynamic applications due to motion blur and errors; therefore it is only suitable where the scene is static. 
If the light source and receiver gain modulation waveform shapes are known (thus each harmonic's amplitude and phase are known), then the error can be predicted in advance in the same manner used to generate the plot in figure 3 . A correction factor can then be generated, and a look-up table can be used to correct the result of equation (4) to achieve the true phase ${ }^{13}$. In practice this can be achieved by calibrating the image ranging system against targets at various distances $^{12}$. This is a time consuming task, and must be repeated each time an influencing factor changes, which may be as intricate as a change in manufacturing parameters, or as simple as altering the modulation frequency. A change in modulation frequency is required where the scene depth exceeds $c / 2 f_{\bmod }$, as the recovered phase angle is periodic over $2 \pi$ radians and leads to an ambiguous range measurement. By using multiple modulation frequencies, the resultant range ambiguity can be removed ${ }^{14}$.

\section{HARMONIC REJECTION SAMPLING}

A unique sampling method has been developed which is capable of rejecting a predetermined number of interfering harmonics during the data acquisition process ${ }^{15}$. This is a preferable solution as it removes the influence of the harmonics during data acquisition, rather than attempting to correct for it through calibration and post processing. The acquisition time also remains unchanged using the new sampling method.

Under normal operation, data are acquired by integrating the signal over a relatively long period. The long integration time improves the measurement SNR, but is primarily due to the limited data read out speed of the CCD/CMOS array (a typical video frame rate is $30 \mathrm{fps}$ ). If the phase of the illumination modulation waveform is shifted during the integration period, then the resultant waveform is an averaged ratio of the waveform before and after the phase shift occurred.

Figure 4 shows a square modulation waveform, which has been shifted twice by $45^{\circ}$, and combined using a ratio $1: \sqrt{2}: 1$. The ratio is achieved by varying the integration time at each phase step relative to the total frame integration time, where the values are selected to result in a quantized approximation to a sinusoid. The resultant effective waveform over the camera integration period is shown in the lower half of figure 4 . The frequency spectrum of the new signal is shown in figure 5, and clearly illustrates that the third and fifth order harmonics have been cancelled.
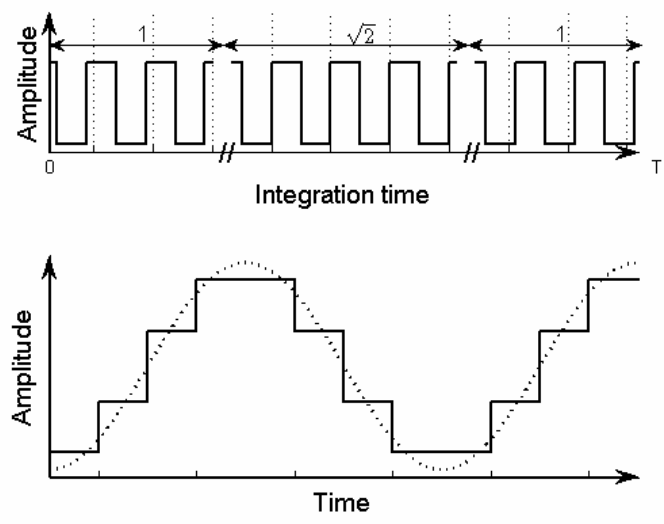

Fig. 4. Harmonic rejection sampling waveform generation. Upper: Original waveform phase stepped twice by $45^{\circ}$; Lower: Resultant effective waveform after integration is a quantized approximation to a sinusoid. 


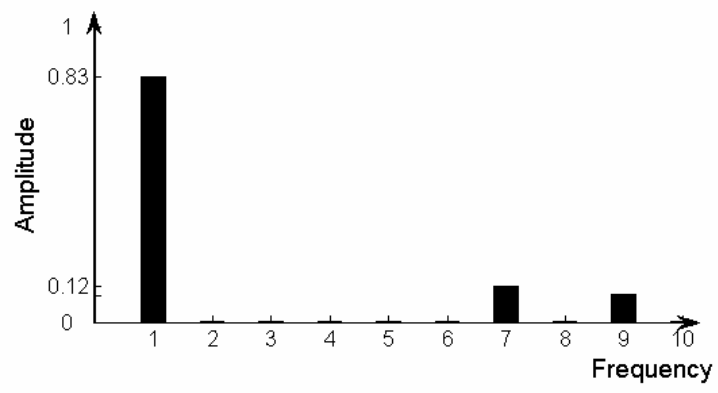

Fig. 5. Frequency spectrum of resultant waveform generated using $45^{\circ}$ steps during the integration period.

To understand the harmonic rejection, the square modulation waveform can be expanded using a Fourier series. The components up to the fifth order are given in equations (7)-(9), where summation using the ratio $1: \sqrt{2}: 1$ results in total cancellation of the third and fifth harmonics.

$$
\begin{aligned}
& f_{1}(t)=\frac{4}{\sqrt{2} \pi}\left[\sin (\omega t)+\cos (\omega t)-\frac{1}{3}(\sin (3 \omega t)-\cos (3 \omega t))-\frac{1}{5}(\sin (5 \omega t)+\cos (5 \omega t))\right] \\
& f_{2}(t)=\frac{4}{\pi}\left[\sin (\omega t)+\frac{1}{3} \sin (3 \omega t)+\frac{1}{5} \sin (5 \omega t)\right] \\
& f_{3}(t)=\frac{4}{\sqrt{2} \pi}\left[\sin (\omega t)-\cos (\omega t)-\frac{1}{3}(\sin (3 \omega t)+\cos (3 \omega t))-\frac{1}{5}(\sin (5 \omega t)-\cos (5 \omega t))\right]
\end{aligned}
$$

A similar technique has been applied to digital RF mixers, where the circuit simultaneously combines three phase shifted waveforms from three separate mixers producing the complex modulation waveform ${ }^{16}$. This is in contrast to the method here where the square wave modulation is retained (requiring little or no change to the existing electronic hardware) and the complex waveform is created by phase stepping the modulation waveform during the frame integration time. As stated previously, each harmonic must occur in both the light source waveform and the receiver gain modulation waveform to be present in the resultant data - now that the effective illumination modulation waveform does not contain these harmonics they are not present in the acquired data.

Applying this sampling method to the simulation above where both the light and receiver modulation waveforms are square, the peak to peak error is reduced from $147 \mathrm{mrad}$ to $16 \mathrm{mrad}$; a reduction of almost an order of magnitude. The error plot is shown in figure 6, and similar to before, contains a distinctive cyclic response, although this time at twice the angular frequency. This error is due to the seventh and ninth order harmonics.

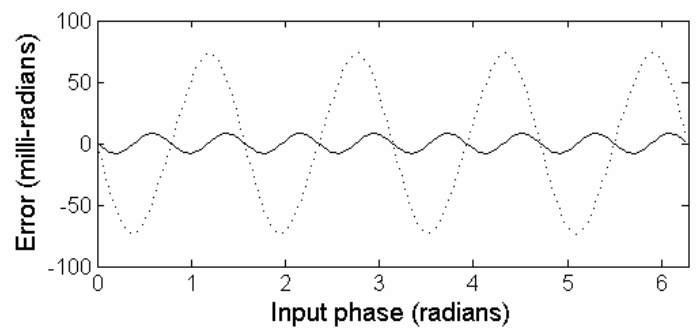

Fig. 6. Error after using the modified sampling scheme with $45^{\circ}$ phase steps (solid line) compared to a standard homodyne system (dashed line).

Repeating the procedure above, using phase steps with a finer resolution of $30^{\circ}$, and an integration time ratio of $1: \sqrt{3}: 2: \sqrt{3}: 1$, the seventh and ninth order harmonics are also suppressed. The errors are further reduced as the first influencing harmonic is eleventh order, which has minimal amplitude. This procedure can be repeated to suppress any number of odd order harmonics, and is only limited by the resolution of the available phase step size, and the resolution of the timer which is responsible for controlling the integration time at each phase step (to generate the correct ratio). 
These simple simulations do not take into consideration noise within the measurements. The amplitude of the fundamental component when utilising this method with phase steps of $45^{\circ}$ reduces to $(2 \sqrt{2}) /(2+\sqrt{2})=0.828$. With finer phase step resolution, the amplitude approaches $\pi / 4=0.785$. Equation (4) itself is not affected by this reduction; however in practical applications the signal to noise ratio (SNR) is reduced resulting in an increase in random error. The range precision of an amplitude modulated laser range finder is given by equation (10), where $f_{\text {mod }}$ is the modulation frequency, and $m$ is the modulation index ${ }^{17}$.

$$
\sigma_{R}=\frac{c}{4 \pi f_{\text {mod }} m S N R}
$$

The modulation index is a measure of the amplitude of the fundamental frequency component divided by the DC offset. A modulation index decrease to 0.785 corresponds to increase in random measurement error by approximately $27 \%$. The systematic errors caused by harmonics are typically much larger than the random errors, so it is likely that this trade off is acceptable. Table 1 gives the modulation index for various illumination and receiver modulation waveforms; it is worth noting that if the light source were amplitude modulated with a sinusoidal waveform instead of a square waveform to remove harmonics as suggested in section 4 , then the same reduction in the modulation index, hence measurement precision, occurs.

Table. 1. Modulation waveform comparison. A larger modulation index leads to smaller random errors. The presence of harmonics can create a systematic error, reducing the measurement accuracy.

\begin{tabular}{|c|c|c|c|}
\hline $\begin{array}{c}\text { Illumination } \\
\text { waveform }\end{array}$ & $\begin{array}{c}\text { Receiver } \\
\text { waveform }\end{array}$ & $\begin{array}{c}\text { Modulation } \\
\text { index }\end{array}$ & Harmonics \\
\hline Square & Square & 0.81 & Present \\
\hline Sine & Square & 0.64 & None \\
\hline Sine & Sine & 0.50 & None \\
\hline Square & $\begin{array}{c}\text { Square } \\
\text { (Harmonic } \\
\text { Rejection) }\end{array}$ & 0.64 & None \\
\hline
\end{tabular}

\section{EXPERIMENTAL RESULTS}

To experimentally verify the harmonic rejection modulation principle, an existing range imaging system ${ }^{14}$ was reconfigured with a new signal generator. The system was configured as shown in figure 7. A diverging laser light source was amplitude modulated at a frequency of $66.67 \mathrm{MHz}$, illuminating a flat panel object. The reflected light is collected by a focusing lens onto the input window of an image intensifier. The photocathode voltage of the image intensifier is also modulated at $66.67 \mathrm{MHz}$, producing gain modulation at this frequency. The output of the image intensifier is coupled via a relay lens to a digital CCD video camera (Dalsa Pantera TF 1M60), which delivers the images to a PC at $25 \mathrm{fps}$. The camera was configured to use 8 by 8 binning to improve the SNR for this experiment, producing an image of $128 \times 128$ pixels.

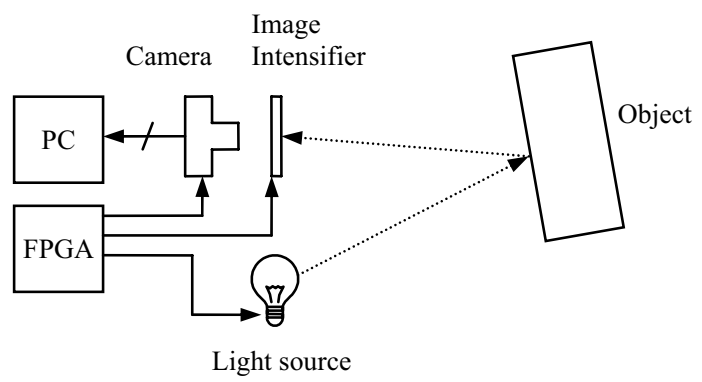

Fig. 7. Experimental configuration.

The two high frequency modulation signals, and a frame trigger for the camera, were produced with an Altera Stratix II FPGA. A phase locked loop (PLL) within the FPGA can generate multiple outputs, and the phase of each output can be reconfigured dynamically while the PLL is running. Using this feature, the $66.67 \mathrm{MHz}$ signal to the light source remained at a fixed phase, while the phase of the signal to the image intensifier was advanced. One concern with using a 
PLL is its inherent phase noise; however since both outputs are generated by the same PLL, any phase noise is common to the outputs and does not contribute any error to the results. The camera frame integration time was divided by the required ratio (e.g. $1: \sqrt{2}: 1$ for phase steps of $45^{\circ}$ ), and a counter within the FPGA is used to trigger the camera integration period and advance the image intensifier signal phase at the required times. Data acquisition consists of capturing four image frames where the image intensifier phase had been advanced by $90^{\circ}$ for each frame. Using equations (2) and (4), the signal amplitude and phase respectively are calculated for each pixel in the image.

A more traditional method of measuring system linearity by physically moving the distance to the object for the next capture was substituted with an electronic approach of introducing delays of $125 \mathrm{ps}$ to the laser signal from the FPGA. This allows the effect of harmonics on equation (4) to be investigated while any other conditions which may influence the error are held constant. The most notable consequence here is that the reflected light intensity remained constant as additional delays are introduced; instead of decreasing by an inverse square with distance which would change the signal to noise ratio (SNR). A stationary object also cancels out any errors due to lens distortion and CCD pixel variation as the object always remains at the focal point and the same pixel(s) can be used during analysis.

\subsection{Linearity}

Data were captured using the traditional homodyne method in which the phase of the image intensifier signal remains constant during the frame integration time. A region of $5 \times 5$ pixels was averaged (to enhance SNR), and the phase calculated using equation (4). The error between the input phase (controlled using the introduced $125 \mathrm{ps}$ delays) and the measured phase is shown in figure 8 . The experiment was repeated five times, and a line has been plotted through the mean error value.

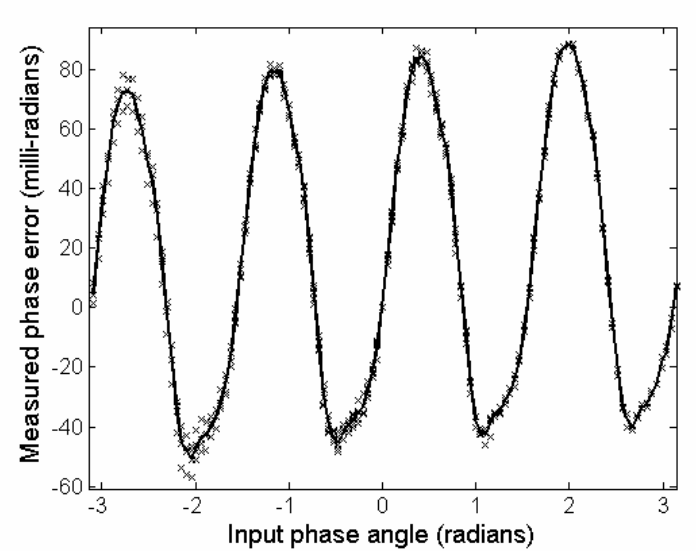

Fig. 8. Measured phase error in a traditional homodyne system. The $3^{\text {rd }}$ and $5^{\text {th }}$ order harmonics are responsible for the evident oscillation.

The measured error in figure 8 closely resembles that predicted in figure 3, with a distinct oscillation at a frequency of $4 \omega$. The peak-to-peak amplitude is approximately $125 \mathrm{mrad}$, which is slightly less than that predicted (147 mrad). This is due to the fact that the laser output waveform and image intensifier gain modulation waveform are not perfect square waves, in this case reducing the amplitude of the $3^{\text {rd }}$ and $5^{\text {th }}$ harmonics, thereby reducing the amplitude of the error. A linear variation occurs from left to right across figure 8 which is most probably due to the image intensifier (and driver circuit) heating during each acquisition sequence. The equipment used is unable to sustain extended periods of operation (required to reach thermal equilibrium) so this trend is visible in all acquisition sequences.

The same experiment was repeated, this time applying the harmonic rejection method described above. The image intensifier signal was phase stepped in increments of $45^{\circ}$ during the CCD integration period, with the resultant error shown in figure 9. The peak-to-peak error has reduced significantly from approximately $125 \mathrm{mrad}$ to $16 \mathrm{mrad}$. The principal systematic error (ignoring the linear trend) is now due to the $7^{\text {th }}$ and $9^{\text {th }}$ order harmonics, which contribute an error with angular frequency $8 \omega$ matching that predicted in figure 6 .

Repeating the experiment using finer resolution phase steps of $30^{\circ}$ during the integration period, the peak-to-peak error is reduced to approximately $12 \mathrm{mrad}$ as illustrated in figure 10. Again the angular frequency component of the error has increased (to $12 \omega$ ), and is now due to the $11^{\text {th }}$ and $13^{\text {th }}$ order components. 


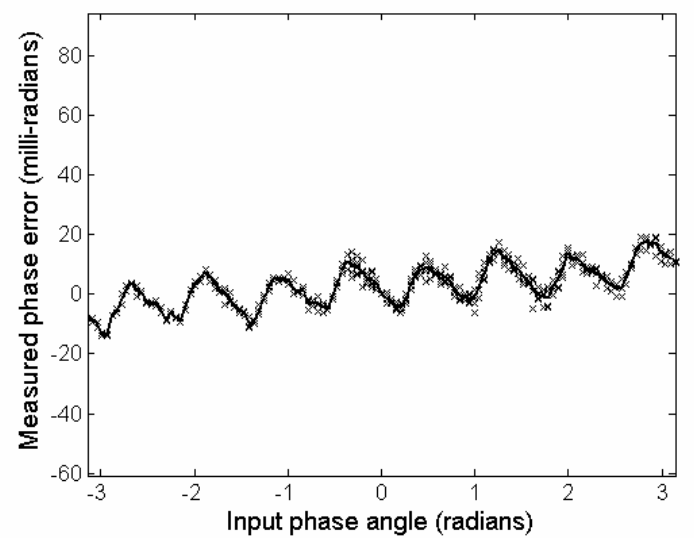

Fig. 9. Measured phase error using the harmonic rejection method, using phase steps of $45^{\circ}$. The error due to $3^{\text {rd }}$ and $5^{\text {th }}$ harmonics is cancelled.

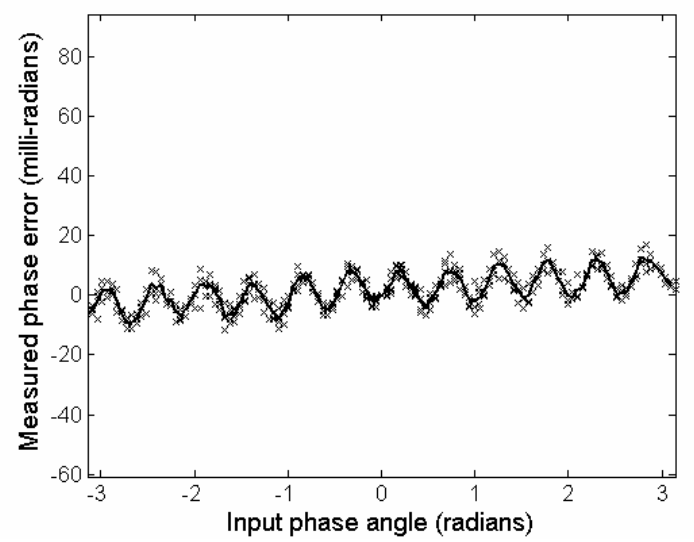

Fig. 10. Measured phase error using the harmonic rejection method, using phase steps of $30^{\circ}$. The error due to $3^{\text {rd }}, 5^{\text {th }}, 7^{\text {th }}$ and $9^{\text {th }}$ order harmonics are cancelled.

\subsection{Precision}

To determine the change in SNR between the traditional homodyne method and the harmonic rejection method, the precision of the phase measurement of each pixel was measured. An acquisition was performed using the homodyne method, capturing 120 samples of the illumination waveform. This large number of samples prevents any significant harmonics from being aliased onto the fundamental signal (as described in section 4), and allows an accurate measurement of the scene due to the high resultant SNR. The acquisition time is 30 times longer than the four frame method, and therefore this technique is only suitable for a static scene. The orientation of the flat plane in the scene is measured from the captured data, providing a high precision reference measurement.

The flat plane object was again measured, this time using the standard four frame homodyne method and the four frame harmonic rejection method. The resultant measurement was segmented into regions of $5 \times 5$ pixels, and an offset added to each region so that the object was aligned with the reference measurement (to compensate for the systematic linearity error in the homodyne method). The variation of each pixel about the reference provides a measure of the system precision. The received signal intensity is not only dependent on the modulation scheme used, but also lighting and reflectivity within the scene, so the mean signal amplitude of each $5 \times 5$ pixel region is also measured. Figure 11 shows the standard deviation of the error for each region plotted against its amplitude, with an inverse square-root fit through the measured points of each method. The measurement precision for the harmonic rejection method is approximately $25 \%$ worse than that of the homodyne method, and is in agreement with the predicted change of $27 \%$ due to the reduction of the modulation index value in equation (10). 


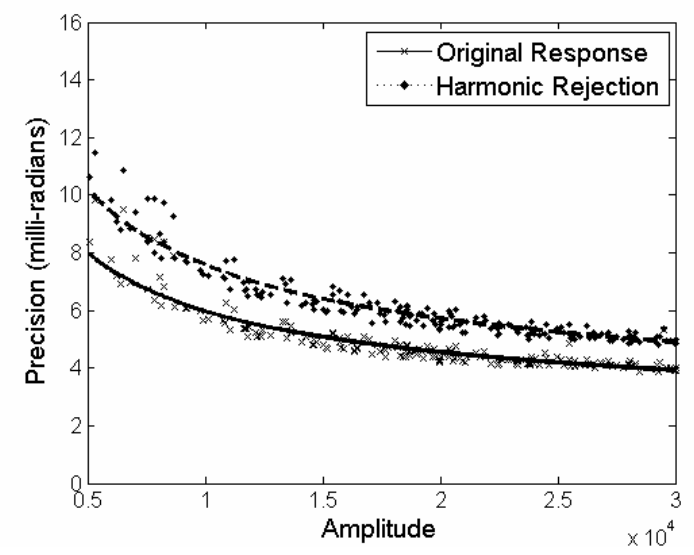

Fig. 11. One-sigma precision measurement for the traditional homodyne method and the harmonic rejection method.

\section{DISCUSSION}

The experimental results closely match those predicted despite the basic assumption that the laser amplitude modulation and image intensifier gain waveforms were square. This reinforces the suitability of this method for any modulation waveform shape, and that the only prerequisite required is to determine the minimum number of harmonics to cancel. It follows that by simply canceling an arbitrarily large number of harmonics, the waveform shape does not need to be known in advance.

Increasing the phase step resolution requires an increase in the system clock frequency of the signal generator electronics. Practicalities may limit this clock speed, thereby inhibiting cancellation of all harmonics present in a given waveform. Figures 8 and 9 demonstrate that by reducing the $90^{\circ}$ phase step size used in a homodyne system to $45^{\circ}$ (which requires doubling the system clock), and applying the harmonic rejection technique, results in an improvement in accuracy of almost 8 times for the modulation waveforms used during our experiment. The harmonic rejection method is therefore beneficial even in its most simplistic form.

It is important for full field range measurement systems to be both accurate and precise. From the results presented in figures $8-11$, the performance of the range measurement system using the homodyne technique is expected to have a one-sigma precision of $2 \mathrm{~mm}$ (5 mrad), and an RMS accuracy of $16 \mathrm{~mm}(44 \mathrm{mrad})$, where the distance values are calculated using equation (1) with the modulation frequency of $66.67 \mathrm{MHz}$. This compares to a precision of $2.5 \mathrm{~mm}$ $(7 \mathrm{mrad})$ and accuracy of $4 \mathrm{~mm}(12 \mathrm{mrad})$ for the harmonic rejection technique with $30^{\circ}$ phase shift resolution. Although precision suffers using the harmonic rejection technique, the loss is minimal compared with the improvement in accuracy.

The error due to the $11^{\text {th }}$ and $13^{\text {th }}$ harmonics is still present in figure 10; hence future work will focus on increasing the phase shift resolution, with the aim of canceling an arbitrarily high number of harmonics (e.g. greater than $50^{\text {th }}$ order) to ensure no influence is present in the range measurement linearity. Theoretically it is possible to calibrate the homodyne system to improve the accuracy while maintaining superior precision over the harmonic rejection technique, but as previously mentioned this is a time consuming task which must be repeated whenever an influencing factor changes.

\section{CONCLUSION}

The unique sampling method described has been shown to reduce the systematic errors due to harmonics in a full field image ranging system. This improvement in performance is obtained with a new modulation signal generation system, while leaving the modulation drive electronics unchanged. The uncalibrated measurement linearity is dependent on the harmonic content of the modulation signals of a particular system. The experimental results shown here suggest that in the presence of harmonics, an improvement in accuracy by an order of magnitude is not unrealistic using the new technique. It was also shown that this is achieved at the expense of measurement precision; however as the original homodyne system provides high precision and low accuracy this trade-off is acceptable, resulting in a system producing both high measurement precision and measurement linearity. 


\section{REFERENCES}

1 S. Christie, S. L. Hill, B. Bury, J. O. Gray, and K. M. Booth, "Design and Development of a Multi-Detecting 2Dimensional Ranging Sensor," Measurement Science \& Technology, vol. 6, pp. 1301-1308, 1995.

2 G. J. Iddan and G. Yahav, "3-D imaging in the studio and elsewhere," Proc. SPIE, vol. 4298, pp. 48-55, 2001.

3 M. Kawakita, K. Iizuka, H. Nakamura, I. Mizuno, T. Kurita, T. Aida, Y. Yamanouchi, H. Mitsumine, T. Fukaya, H. Kikuchi, and F. Sato, "High-definition real-time depth-mapping TV camera: HDTV axi-vision camera," Optics Express, vol. 12, pp. 2781-2794, 2004.

4 W. Schroeder, E. Forgber, and S. Estable, "Scannerless laser range camera," Sensor Review, vol. 19, pp. 285-291, 1999.

5 R. O. Nellums, R. D. Habbit, M. R. Heying, T. A. Pitts, and J. V. Sandusky, "3D scannerless LADAR for Orbiter inspection," Proc. SPIE - Spaceborne Sensors III, vol. 6220, pp. 62200G, 2006.

6 T. Spirig, M. Marley, and P. Seitz, "The multitap lock-in CCD with offset subtraction," IEEE Transactions on Electron Devices, vol. 44, pp. 1643-1647, 1997.

7 R. Lange, P. Seitz, A. Biber, and S. Lauxtermann, "Demodulation Pixels in CCD and CMOS Technologies for Time-of-Flight Ranging," Proc. SPIE, vol. 3965, pp. 177-189, 2000.

8 R. Schwarte, "Breakthrough in multichannel laser-radar technology providing thousands of high-sensitive lidar receivers on a chip," Proceedings of SPIE, vol. 5575, pp. 126-136, 2004.

9 T. Oggier, M. Lehmann, R. Kaufmann, M. Schweizer, M. Richter, P. Metzler, G. Lang, F. Lustenberger, and N. Blanc, "An all-solid-state optical range camera for 3D real-time imaging with sub-centimeter depth resolution (SwissRanger)," Proc. SPIE - Optical Design and Engineering, vol. 5249, pp. 534-545, 2004.

10 S. Hsu, S. Acharya, A. Rafii, and R. New, "Performance of a Time-of-Flight Range Camera for Intelligent Vehicle Safety Applications," Advanced Microsystems for Automotive Applications 2006, pp. 205-214, 2006.

11 S. B. Gokturk, H. Yalcin, and C. Bamji, "A Time-Of-Flight Depth Sensor - System Description, Issues and Solutions," Proc. 2004 IEEE Conf. Computer Vision and Pattern Recognition Workshop, pp. 35, 2004.

12 T. Kahlmann and H. Ingensand, "Calibration of the fast range imaging camera SwissRanger for use in the surveillance of the environment," Proceedings of SPIE, vol. 6396, pp. 639605, 2006.

13 R. Lange, "3D Time-of-flight distance measurement with custom solid-state image sensors in CMOS/CCDtechnology," Ph.D. dissertation, University of Siegen, 2000.

14 A. A. Dorrington, M. J. Cree, A. D. Payne, R. M. Conroy, and D. A. Carnegie, "Achieving sub-millimetre precision with a solid-state full-field heterodyning range imaging camera," Meas. Sci. Technol., vol. 18, pp. 2809-2816, 2007.

15 A. D. Payne, "Harmonic rejection sampling methodology," New Zealand Patent Application No. 562739, 2007

16 J. A. Weldon, R. S. Narayanaswami, J. C. Rudell, L. Lin, M. Otsuka, S. Dedieu, L. Tee, K. C. Tsai, C. W. Lee, and P. R. Gray, "A 1.75-GHz highly integrated narrow-band CMOS transmitter with harmonic-rejection mixers," Ieee Journal of Solid-State Circuits, vol. 36, pp. 2003-2015, 2001.

17 A. Jelalian, Laser Radar Systems: Artech House, Boston, 1992. 